\title{
The role of the diffusive protons in the gamma-ray emission of SNR RX J1713.7-3946
}

\author{
Xiao Zhang ${ }^{1}$ and Yang Chen ${ }^{1,2}$ \\ ${ }^{1}$ Department of Astronomy, Nanjing University, 163 Xianlin Avenue, Najing 210023, China \\ email: xiaozhang@nju.edu.cn \\ ${ }^{2}$ Key Laboratory of Modern Astronomy and Astrophysics, Nanjing University, Ministry of \\ Education, Najing 210023, China \\ email: ygchen@nju.edu.cn
}

\begin{abstract}
RX J1713.7-3946 is a prototype in the $\gamma$-ray-bright supernova remnants (SNRs) and is in continuing debates on its hadronic versus leptonic origin of the $\gamma$-ray emission. We explore the role played by the diffusive relativistic protons that escape from the SNR shock wave in the $\gamma$-ray emission, apart from the emission of high energy particles from the inside of the SNR. In the scenario that the SNR shock propagates in a clumpy molecular cavity, we consider that the $\gamma$-ray emission from the inside of the SNR may either arise from the IC scattering or from the interaction between the trapped energetic protons and the shocked clumps. The dominant origin between them depends on the electron-to-proton number ratio. The surrounding molecular cavity wall is considered to also produce $\gamma$-ray emission due to the "illumination" by the diffusive protons that escaped from the shock wave during the expansion history. The broadband spectrum can be well explained by this two-zone model, in which the $\gamma$-ray emission from the inside governs the $\mathrm{TeV}$ band, while the outer emission component substantially contributes to the GeV $\gamma$-rays. The two-zone model can also explain the $\mathrm{TeV} \gamma$-ray radial brightness profile that significantly stretches beyond the nonthermal X-ray emitting region.
\end{abstract}

Keywords. radiation mechanisms: nonthermal, ISM: individual (G347.3-0.5), (ISM:) supernova remnants, gamma rays: theory

\section{Introduction}

SNR RX J1713.7-3946 (G347.3-0.5) was discovered by ROSAT X-ray observation (Pfeffermann \& Aschenbach 1996) and suggested to be the remnant of the historical supernova AD393 (Wang et al. 1997, hereafter an age of the remnant $t_{\text {age }} \sim 1620 \mathrm{yr}$ will be used). Its shell-like X-ray emission is dominated by non-thermal component, lack of thermal line features (e.g., Koyama et al. 1997; Slane et al. 1999; Cassam-Chenaï et al. 2004). It is found to be confined in a molecular cavity and the elevated gas temperature and broad molecular line wings in a few molecular cloudlets are ascribed to the highenergy events of the SNR (Fukui et al. 2003; Moriguchi et al. 2005). In radio band, it shows faint emission and has an average angular $60^{\prime}$ in diameter, corresponding to an average radius $R_{s} \sim 9 \mathrm{pc}$ at distance $d \approx 1 \mathrm{kpc}$ (e.g. Fukui et al. 2003; Moriguchi et al. 2005). The SNR is suggested to be in the free expansion evolutionary phase (e.g. Moriguchi et al. 2005; Sano et al. 2010), and that the forward shock has not yet reached the wall enclosing the wind-blown cavity in view of the high velocity $\left(V_{s} \sim 4000 \mathrm{~km} \mathrm{~s}^{-1}\right)$ currently measured (Federici et al. 2015). 
Since the detection of $\mathrm{TeV} \gamma$-ray emission from it (Enomoto et al. 2002; Aharonian et al. 2004, 2007), SNR RX J1713.7-3946 has absorbed enormous amount of attention and aroused constant debates on its hadronic versus leptonic origin of the $\gamma$-ray emission (e.g. Aharonian et al. 2006; Berezhko \& Völk 2008; Liu et al. 2008; Morlino et al. 2009; Zirakashvili \& Aharonian 2010). The debates seemed to have been concluded when the 2-yr Fermi-LAT observation revealed a hard GeV spectrum with a power-law photon index $\Gamma=1.5 \pm 0.1$ (Abdo et al. 2011), which appears to support the leptonic scenario (e.g., Abdo et al. 2011; Yuan et al. 2011; Li et al. 2011; Ellison et al. 2012; Lee et al. 2012; Finke \& Dermer 2012). Subsequently, however, a strong correlation of azimuthal distribution was found between the $\mathrm{TeV} \gamma$-ray flux and the column density of total interstellar protons, which is in favor of hadronic origin of the $\gamma$-rays (Fukui et al. 2012). Some hadronic interaction models have also been elaborated to interpret the hard $\mathrm{GeV}$ emission (Inoue et al. 2012; Gabici \& Aharonian 2014; Federici et al. 2015). Both the scenarios in terms of relativistic leptons and protons accelerated via standard DSA can explain the hard spectrum with their advantages, but meanwhile also leave difficulties, respectively.

Here we explore the role played by the diffusive relativistic protons that escape from the SNR shock and hit the surrounding dense gas in the $\gamma$-ray emission of RX J1713.7-3946, considering that the blast wave is propagating in a molecular cavity, in which there may be an accumulation of shocked clumps. In the following, we show that the GeV-TeV $\gamma$ ray spectrum of RX J1713.7-3946 with updated 5-yr Fermi-LAT data can be perfectly interpreted with a two-zone scenario, in which the $\pi^{0}$-decay $\gamma$-rays resulted from the diffusive protons will substantially contribute to the $\mathrm{GeV} \gamma$-rays while the leptonic process or the p-p interaction in the shocked clumps will be responsible for the $\mathrm{TeV} \gamma$-rays.

\section{A two-zone Model}

Considering the large shock velocity and the low ambient density, we assume that the shock of SNR RX J1713.6-3946 doesn't extensively hit the surrounding MC and is still evolving in the molecular cavity, but may sweep up some dense clumps (see Figure 1). The first (outer) emission zone is the cavity wall (the surrounding dense matter at the cavity boundary, which may be clumpy even without a sharp interface), which is bombarded by the protons that escaped from the shock wave during the history of expansion. The second (inner) emission zone is inside the SNR, and the emission could be either IC scattering off the accelerated electrons or the hadronic emission from the shocked clumps. Due to the two possible channels of generating $\gamma$-rays inside SNR, two cases will be considered within inner zone: (I) it is dominated by the IC process of the accelerated electron; and (II) it is dominated by the collision of the accelerated protons with the shocked clumps. For comparison, we also only use the inner zone to fit the broadband data (referred to as Model Ib and Model IIb respectively). All models are summarized in Figure 1.

Distribution of particles in the inner zone. The shock accelerated particles are assumed to obey a power-law distribution with an high energy cut-off:

$$
d N_{i} / d E_{i}=A_{i} E_{i}^{\alpha_{i}} \times \exp \left(-E_{i} / E_{\mathrm{c}, i}\right)
$$

where $i=\mathrm{e}, \mathrm{p}, E_{i}$ is the particle kinetic energy, $\alpha_{i}$ is the power-law spectral index and is taken as $\alpha_{\mathrm{e}}=\alpha_{\mathrm{p}}=\alpha$, and $E_{\mathrm{c}, i}$ is the cut-off energy and will be set to the CR "knee" energy for the protons, leaving $E_{\mathrm{c}, \mathrm{e}}$ as free parameter. The normalization parameter $A_{i}$ is determined from the total kinetic energy of particles above $1 \mathrm{GeV}$. In our calculation, two physical parameters are adopted instead of the normalization parameters: the energy conversion efficiency $\eta$, namely the faction of explosion energy 


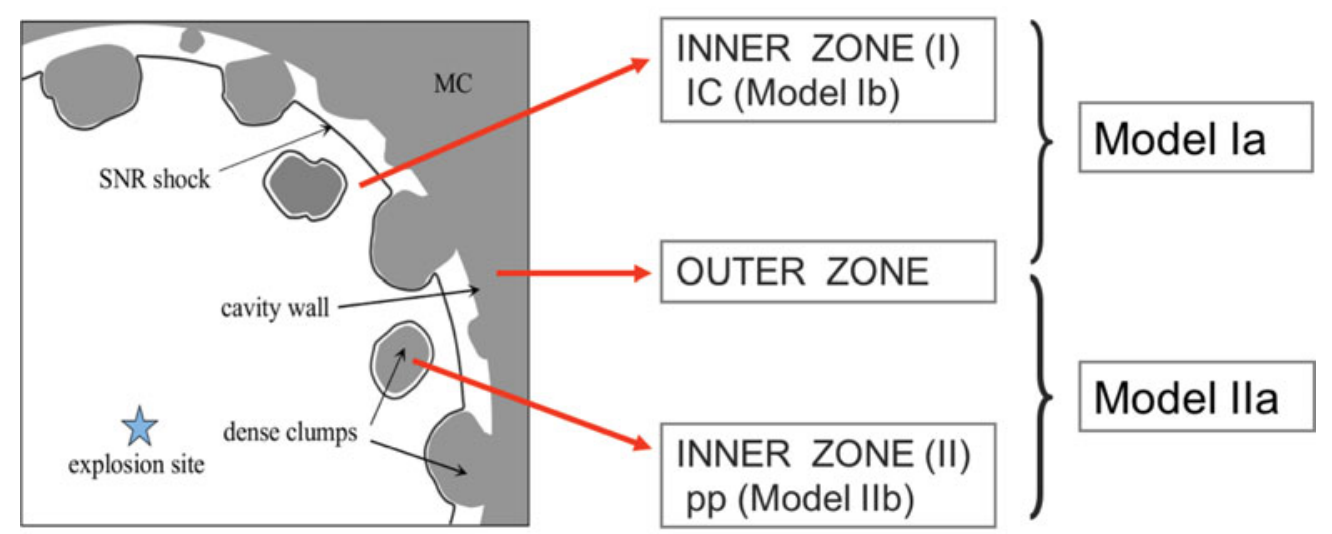

Figure 1. Schematic of the two-zone model.

converted to $\mathrm{CR}$ energy, and the number ratio between the accelerated electrons and protons at $1 \mathrm{GeV}, K_{\mathrm{ep}}=A_{\mathrm{e}} / A_{\mathrm{p}}$.

Distribution of particles in the outer zone. For the continuous injection of the accelerated protons that escape from the propagating shock wave surface, Li \& Chen (2010) established an "accumulative diffusion" model, in which the protons at given position is a collection of the diffusive protons escaping from the entire shock surface at different radii throughout the history of the SNR expansion. Instead of the treatment in Li \& Chen (2010), the distribution function of the escaping protons can be simplified by directly solving the diffusion equation of the escaping protons as:

$$
f\left(E_{\mathrm{p}}, R, t\right)=\frac{\int_{0}^{t} \frac{Q_{\mathrm{p}}\left(E_{\mathrm{p}}\right)}{4 \pi^{3 / 2} R_{s}\left(t_{i}\right) R_{\mathrm{d}} R}}{\left\{\exp \left[-\left(\frac{R-R_{s}\left(t_{i}\right)}{R_{\mathrm{d}}}\right)^{2}\right]-\exp \left[-\left(\frac{R+R_{s}\left(t_{i}\right)}{R_{\mathrm{d}}}\right)^{2}\right]\right\} \mathrm{d} t_{i}}
$$

where $f\left(E_{\mathrm{p}}, R, t\right)$ is the distribution of the escaping protons with energy $E_{\mathrm{p}}$ at a given distance $R$ from the SNR center at a given time $t$ from the explosion time, $Q_{\mathrm{p}}\left(E_{\mathrm{p}}\right)$ is the injection rate from the spherical surface $4 \pi R_{s}^{2}, R_{s}(t)$ is the shock radius, and $R_{\mathrm{d}}=2 \sqrt{D\left(t-t_{i}\right)}$ is diffusion radius. Here, we assume that the diffusion coefficient has the form of $D\left(E_{\mathrm{p}}\right)=10^{28} \chi\left(E_{\mathrm{p}} / 10 \mathrm{GeV}\right)^{\delta} \mathrm{cm}^{2} \mathrm{~s}^{-1}$, where $\chi$ is the correction factor of slow diffusion around SNR, and $\delta \approx 0.3-0.7$ (Berezinskii 1990) is the index of diffusion coefficient.

Mass of the gas in the inner zone II. Following Inoue et al. (2012), the total mass of the gas in the clumps directly bombarded by the accelerated protons, $m_{c, t o t}$, can be obtained via three steps: (1) Estimate the average mass density $\rho_{c}$ for each clump with physical parameters given by Sano et al. (2013, see Table 3 and Table 4 therein). (2) Estimate the mass in the outer layer of each clump that is penetrated by relativistic protons using $m_{c}=4 \pi \rho_{c} a_{c}^{2} l_{\mathrm{pd}}$ (see Inoue et al. 2012), where $a_{c}$ is the clump radius and $l_{\mathrm{pd}} \simeq \sqrt{\kappa_{d} t_{\mathrm{pd}}}$ is the penetration depth of the accelerated protons into the clumps during diffusion time $t_{\mathrm{pd}}$ with the diffusion coefficienct $\kappa_{d}=4 \eta_{\mathrm{B}} c E_{\mathrm{p}} / 3 \pi e B_{\mathrm{MC}}\left(\eta_{\mathrm{B}}\right.$ is the degree of magnetic field fluctuations and $B_{\mathrm{MC}}$ is the magnetic field in the dense region of MCs). The diffusion time is estimated as $t_{\mathrm{pd}} \sim 0.2\left(l_{m} / 1 \mathrm{pc}\right)\left(V_{s} / 4000 \mathrm{~km} \mathrm{~s}^{-1}\right)^{-1} \mathrm{kyr}$, where $V_{s}=4000 \mathrm{~km} \mathrm{~s}^{-1}$ (e.g., Cassam-Chenaï et al.2004; Acero et al. 2009) is the shock velocity and $l_{m} \sim 1 \mathrm{pc}$ is the mean length that the shock moves forward from the clumps, estimated from the spatial distribution of the clumps (Sano et al. 2013). (3) Scale the mass of the gas collided by protons according to the area fraction covered by X-rays for 
Table 1. The SED fitted parameters with $1 \sigma$ statistical error.

\begin{tabular}{lccccccccc}
\hline Model & $K_{\mathrm{ep}}$ & $\begin{array}{c}\eta \\
(\%)\end{array}$ & $\alpha$ & $\begin{array}{c}B_{\mathrm{SNR}} \\
(\mu \mathrm{G})\end{array}$ & $\begin{array}{c}E_{\mathrm{c}, \mathrm{e}} \\
(\mathrm{TeV})\end{array}$ & $\begin{array}{c}E_{\mathrm{c}, \mathrm{p}} \\
(\mathrm{TeV})\end{array}$ & $\chi / 0.01$ & $\delta$ & $\begin{array}{c}M_{\mathrm{t}} \\
\left(10^{3} M_{\odot}\right)\end{array}$ \\
\hline Ia & $0.01^{a}$ & $2.5_{-0.9}^{+0.6}$ & $2.09_{-0.04}^{+0.03}$ & $15.9_{-1.3}^{+4.2}$ & $34.2_{-4.0}^{+1.7}$ & - & $1.2_{-0.4}^{+1.0}$ & $0.67_{-0.14}^{+0.03}$ & $8.8_{-3.1}^{+6.6}$ \\
Ib & $0.01^{a}$ & $4.1_{-0.5}^{+0.5}$ & $2.11_{-0.02}^{+0.02}$ & $12.7_{-0.3}^{+0.3}$ & $38.6_{-0.8}^{+0.7}$ & - & - & - & - \\
IIa & $0.001^{a}$ & $6.0_{-3.3}^{+2.9}$ & $2.09_{-0.06}^{+0.08}$ & $37.9_{-5.5}^{+6.2}$ & $22.2_{-5.0}^{+2.9}$ & $81.3_{-11.9}^{+13.7}$ & $3.1_{-0.4}^{+0.7}$ & $0.55_{-0.25}^{+0.15}$ & $2.6_{-1.0}^{+1.8}$ \\
IIb & $0.001^{a}$ & $10.6_{-1.5}^{+1.8}$ & $2.10_{-0.03}^{+0.02}$ & $27.6_{-2.0}^{+1.5}$ & $26.2_{-0.8}^{+1.0}$ & $60.4_{-10.1}^{+08.8}$ & - & - & - \\
\hline
\end{tabular}

Notes:

${ }^{a}$ Fixed in the model fit.

Table 2. The best-fit $\chi^{2}$ value for each set of data.

\begin{tabular}{lccccc}
\hline Model & radio & X-ray & $\mathrm{GeV}$ & $\mathrm{TeV}$ & $\chi_{\nu}^{2}$ (d.o.f.) \\
\hline $\mathrm{Ia}$ & 3.8 & 364.6 & $10.2\left(13.5^{a}\right)$ & 37.1 & $1.78(233)$ \\
$\mathrm{Ib}$ & 0.09 & 374.1 & $36.3\left(5.5^{a}\right)$ & 120.0 & $2.25(236)$ \\
$\mathrm{IIa}$ & 3.7 & 364.6 & $9.1\left(7.0^{a}\right)$ & 36.4 & $1.78(232)$ \\
$\mathrm{IIb}$ & 0.8 & 368.6 & $23.9\left(3.0^{a}\right)$ & 41.7 & $1.85(235)$ \\
\hline
\end{tabular}

Notes:

${ }^{a}$ Corresponding to the old Fermi data (Abdo et al. 2011).

each clump and sum the mass of the clumps. Hence, we obtain

$$
m_{c, t o t} \approx 13\left(\frac{\eta_{\mathrm{B}}}{1}\right)\left(\frac{E_{\mathrm{p}}}{10 \mathrm{GeV}}\right)^{1 / 2}\left(\frac{B_{\mathrm{MC}}}{100 \mu \mathrm{G}}\right)^{-1 / 2}\left(\frac{t_{\mathrm{pd}}}{0.2 \mathrm{kyr}}\right)^{1 / 2} M_{\odot} .
$$

\section{Modelling and Results}

Spectral behavior. We use the two-zone model to fit the broadband spectra of SNR $\mathrm{RX}$ J1713.7-3946 and employ the MCMC approach to constrain the model parameters. The best-fit parameters with $1 \sigma$ statistical uncertainties and the $\chi^{2}$ values are listed in the Table 1 and Table 2 , respectively. The model $\gamma$-ray spectral energy distribution (SED, see Figure 2) shows that this model perfectly explains the $\gamma$-ray data and that the hadronic $\gamma$-rays from the escaping protons play an important role on the explanation of the spectral data, especially in the $\mathrm{GeV}$ band. In the energy range from few $\mathrm{GeV}$ to several $100 \mathrm{GeV}$, the $\gamma$-rays are mainly contributed by the hadronic emission produced within the outer emission zone. Given the presence of the shocked clumps, in the remaining $\gamma$ ray band the dominant mechanism of $\gamma$-ray emission in the downstream depends on the electron-to-proton number ratio, $K_{\mathrm{ep}}$ : it was found that the hadronic emission from the inside clumps dominates for $K_{\mathrm{ep}}<\sim 4 \times 10^{-3}$ while the leptonic component dominates for $K_{\mathrm{ep}}>\sim 4 \times 10^{-3}$. In addition, we also calculate the $\chi^{2}$ value in $\mathrm{GeV}$ band using our best-fit SED and the previous Fermi data (Abdo et al. 2011), which are listed in Table 2 with parentheses.

Spatial behavior. According to the flux ratio between the outer and inner components derived from the above model calculation, we fit the $\mathrm{TeV}$ profile of the sector-shaped "region 3" given in de Naurois (2015) and the entire SNR given in Aharonian et al. (2006), assuming uniform emissivities in the two emission zones. Then, the TeV $\gamma$-ray profile is determined by the positions $\left(R_{s}, R_{w}\right)$ and thicknesses $\left(\Delta R_{s}, \Delta R_{w}\right)$ of the two mission zones. It should be noted that $R_{s}$ represents the outer radius of the inner emission zone while $R_{w}$ is the inner radius of the outer emission zone. In Figure 3, we 

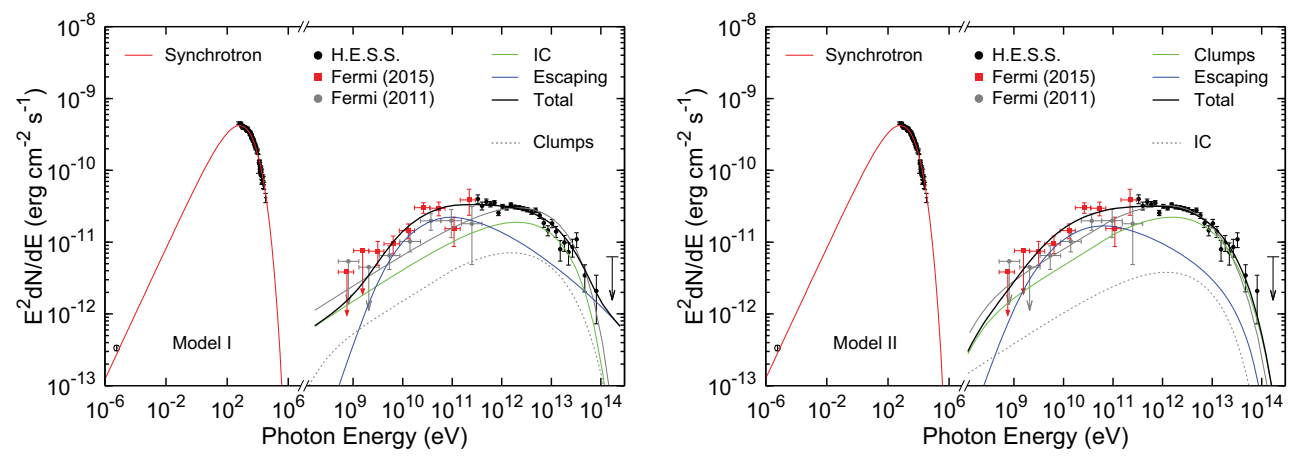

Figure 2. The best fitted broad-band SED of SNR RX J1713.7-3946 according to the radio (Acero et al. 2009), X-ray (Tanaka et al. 2008), GeV (Fermi: Abdo et al. 2011; Federic et al. 2015) and TeV (H.E.S.S.: Aharonian et al. 2011) data for Model Ia (left) and Model IIa (right). The grey solid lines represent the fitting SED for Model Ib (left) and Model IIb (right).

Table 3. The fitted parameters used in Figure 3.

\begin{tabular}{lccccc}
\hline Model & Ratio $^{a}$ & $R_{s}[\mathrm{pc}]$ & $R_{w}\left[R_{s}\right]$ & $\Delta R_{s}\left[R_{s}\right]$ & $\Delta R_{w}\left[R_{s}\right]$ \\
\hline Ia & $4.1: 5.9\left(3.9: 6.1^{b}\right)$ & $7.0\left(9.0^{b}\right)$ & $1.13\left(1.11^{b}\right)$ & $0.12\left(0.23^{b}\right)$ & $0.12\left(0.22^{b}\right)$ \\
IIa & $2.8: 7.2\left(2.7: 7.3^{b}\right)$ & $7.0\left(9.0^{b}\right)$ & $1.20\left(1.11^{b}\right)$ & $0.10\left(0.18^{b}\right)$ & $0.09\left(0.20^{b}\right)$ \\
\hline
\end{tabular}

Notes:

${ }^{a}$ Flux ratio (above $0.25 \mathrm{TeV}$ for "region 3" and between 0.3 and $40 \mathrm{TeV}$ for the entire remnant) between the outer and inner zone derived from the model calculations.

${ }^{b}$ Results in parentheses are corresponding to the entire remnant.
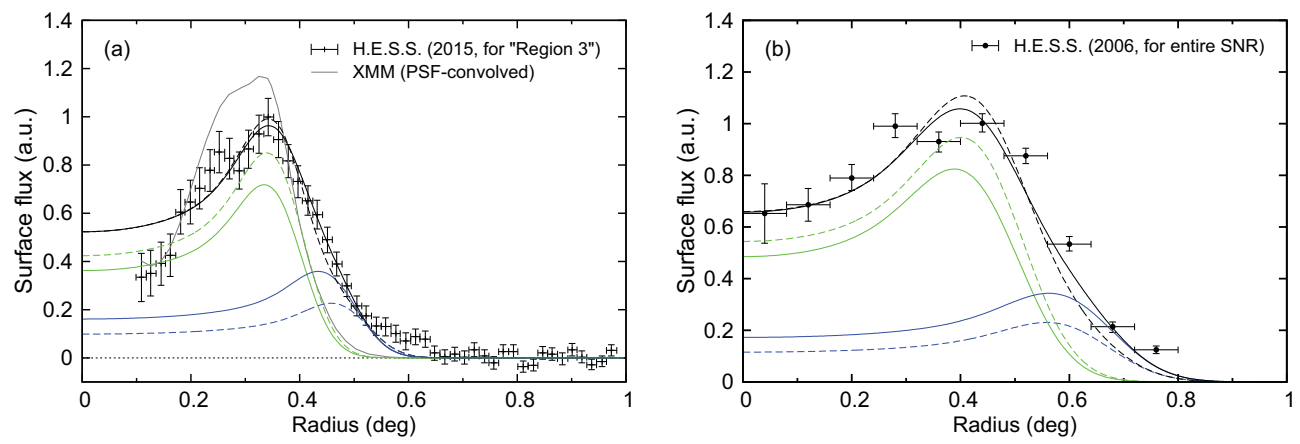

Figure 3. (a) $\mathrm{TeV} \gamma$-ray radial surface brightness profile of sector "region 3". The H.E.S.S. flux data and the XMM-Newton X-ray brightness profile convolved with the H.E.S.S. PSF of $3^{\prime}$ (grey line) are adopted from de Naurois (2015). The radial profile of "region 3" is fitted with a two-zone emission (in black), which is the sum of the inner (in green) and outer (in blue) components. The solid and dashed lines represent the profiles in Model Ia and Model IIa, respectively. All of the fitting profile lines have been convolved with the H.E.S.S. PSF of $3^{\prime}$. (b) $\mathrm{TeV} \gamma$-ray radial surface brightness profile for the entire remnant. The H.E.S.S. flux data are adopted from Aharonian et al. (2006). The meanings of all lines convolved with the H.E.S.S. PSF of $5^{\prime}$ are the same as those in (a).

display the fitting profile lines (black) which are the sum of the inner (green) and outer (blue) components can explain the observational data. For example, the profile lines in left pannel can reproduce both the $\mathrm{TeV}$ brightness peak, which is coincident with the non-thermal X-ray peak, and the broad profile wing that extends outside the X-ray 
emitting region. For the entire remnant, the fitting profiles match the observational data at well. All parameters used to plot Figure 3 can be found in Table 3 .

\section{Summary}

In the scenario that the SNR shock is propagating in a molecular cavity, we have explored the role played by the diffusive relativistic protons that escaped from the SNR shock wave in the $\gamma$-ray emission, apart from the emission of high energy particles from the inside of the SNR. The broad-band fluxes can be well explained by the two-zone model, in which the $\gamma$-ray emission from the inside governs the $\mathrm{TeV}$ band, while the outer emission component substantially contributes to the $\mathrm{GeV} \gamma$-rays. Meantime, we show that the two-zone model can also reproduce the $\mathrm{TeV} \gamma$-ray radial brightness profile that significantly extends outside the nonthermal X-ray emitting region.

\section{References}

Abdo, A. A., Ackermann, M., Ajello, M., et al. 2011, ApJ, 734, 28

Acero, F., Ballet, J., Decourchelle, A., et al. 2009, A\& A, 505, 157

Aharonian, F., Akhperjanian, A. G., Bazer-Bachi, A. R., et al. 2006, A\& A, 449, 223

Aharonian, F., Akhperjanian, A. G., Bazer-Bachi, A. R., et al. 2007, A\& A, 464, 235

Aharonian, F., Akhperjanian, A. G., Bazer-Bachi, A. R., et al. 2011, A\& A, 531, C1

Aharonian, F. A. \& Atoyan, A. M. 1996, A\& A, 309, 917

Aharonian, F. A., Akhperjanian, A. G., Aye, K.-M., et al. 2004, Nature, 432, 75

Berezhko, E. G. \& Völk, H. J. 2008, A\& A, 492, 695

Berezinskii, V. S., Bulanov, S. V., Dogiel, V. A., \& Ptuskin, V. S. 1990, Astrophysics of cosmic rays

Cassam-Chenaï, G., Decourchelle, A., Ballet, J., et al. 2004, A\& A, 427, 199

de Naurois, M. 2015, ArXiv e-prints: 1510.00635

Ellison, D. C., Slane, P., Patnaude, D. J., \& Bykov, A. M. 2012, ApJ, 744, 39

Enomoto, R., Tanimori, T., Naito, T., et al. 2002, Nature, 416, 823

Fan, Z. H., Liu, S. M., Yuan, Q., \& Fletcher, L. 2010, A\& A, 517, L4

Federici, S., Pohl, M., Telezhinsky, I., Wilhelm, A., \& Dwarkadas, V. V. 2015, A\& A, 577, A12

Finke, J. D. \& Dermer, C. D. 2012, ApJ, 751, 65

Fukui, Y., Moriguchi, Y., Tamura, K., et al. 2003, PASJ, 55, L61

Fukui, Y., Sano, H., Sato, J., et al. 2012, ApJ, 746, 82

Gabici, S. \& Aharonian, F. A. 2014, MNRAS, 445, L70

Inoue, T., Yamazaki, R., Inutsuka, S.-i., \& Fukui, Y. 2012, ApJ, 744, 71

Koyama, K., Kinugasa, K., Matsuzaki, K., et al. 1997, PASJ, 49, L7

Lee, S.-H., Ellison, D. C., \& Nagataki, S. 2012, ApJ, 750, 156

Li, H. \& Chen, Y. 2010, MNRAS, 409, L35

Li, H., Liu, S., \& Chen, Y. 2011, ApJL, 742, L10

Liu, S., Fan, Z.-H., Fryer, C. L., Wang, J.-M., \& Li, H. 2008, ApJL, 683, L163

Moriguchi, Y., Tamura, K., Tawara, Y., et al. 2005, ApJ, 631, 947

Morlino, G., Amato, E., \& Blasi, P. 2009, MNRAS, 392, 240

Pfeffermann, E. \& Aschenbach, B. 1996, in Roentgenstrahlung from the Universe, ed. H. U. Zimmermann, J. Trümper, \& H. Yorke, 267-268

Sano, H., Sato, J., Horachi, H., et al. 2010, ApJ, 724, 59

Sano, H., Tanaka, T., Torii, K., et al. 2013, ApJ, 778, 59

Slane, P., Gaensler, B. M., Dame, T. M., et al. 1999, ApJ, 525, 357

Tanaka, T., Uchiyama, Y., Aharonian, F. A., et al. 2008, ApJ, 685, 988

Wang, Z. R., Qu, Q.-Y., \& Chen, Y. 1997, A\& A, 318, L59

Yuan, Q., Liu, S., Fan, Z., Bi, X., \& Fryer, C. L. 2011, ApJ, 735, 120

Zirakashvili, V. N. \& Aharonian, F. A. 2010, ApJ, 708, 965 